\title{
Linezolid for the treatment of hospital-acquired pneumonia in a Chinese tertiary hospital
}

This article was published in the following Dove Press journal:

ClinicoEconomics and Outcomes Research

9 October 2015

Number of times this article has been viewed

\section{Beth A Lesher' \\ Zhengyin Liu ${ }^{2}$ \\ Yixi Chen ${ }^{3}$ \\ Xin $\mathrm{Gao}^{\prime}$}

'Pharmerit International, Bethesda, MD, USA; ${ }^{2}$ Department of Infectious Disease, Peking Union Medical College Hospital, Chinese Academy of Medical Sciences and Peking Union Medical College, Beijing, People's Republic of China; ${ }^{3}$ Pfizer Investment Co, Ltd, Beijing, People's Republic of China

Correspondence: Beth A Lesher Pharmerit International, 4350 East-West Highway, Suite 430, Bethesda, MD 208I4, USA

Tel + I 2408219665

Fax + I 24082 I I296

Email blesher@pharmerit.com

\section{Dear editor}

We read with interest the study published by Song et al describing their retrospective analysis of the clinical response and hospital costs associated with the empiric use of vancomycin or linezolid therapy in patients with hospital-acquired pneumonia (HAP) in the People's Republic of China. ${ }^{1}$ Results from matched comparisons showed similar clinical response ( $30.0 \%$ vs $31.7 \% ; P=0.847)$ and treatment failure rates $(55.0 \%$ vs $45.0 \%$; $P=0.289$ ) but a significantly lower in-hospital mortality rate ( 3.3 vs $18.3 \% ; P=0.013$ ) for vancomycin-treated patients compared to linezolid-treated patients. Treatment failure rates (odds ratio [OR], 1.139; $P=0.308$ ) and risk of in-hospital mortality were comparable (OR, 0.186; $P=0.055$ ) between vancomycin- and linezolid-treated patients following further adjustment for an imbalance in variables between the matched treatment groups. Reported total hospital costs were not significantly different between vancomycin-(RMB 113,160) and linezolid (RMB 133,825)-treated patients ( $P=0.076)$.

While we applaud these authors for publishing these real-world data, there are several issues that deserve further attention. First, although Song et al used one-to-one propensity score matching to create matched pairs, significant differences remained in baseline characteristics between the linezolid and vancomycin treatment groups. Specifically, patients who received linezolid received less combination therapy compared to those who received vancomycin $(18.3 \%$ vs $43.3 \% ; P=0.007)$ and required more current $(46.7 \%$ vs $16.7 \% ; P=0.001)$ or previous $(63.3 \%$ vs $46.7 \% ; P=0.033)$ mechanical ventilation. More patients treated with vancomycin than linezolid received frontline treatment $(51.7 \%$ vs $23.3 \%$; $P=0.001)$ with cephalosporins whereas more patients treated with linezolid than vancomycin received frontline treatment with other beta-lactams $(56.7 \%$ vs $36.7 \% ; P=0.029)$. This is not a successful matching as several significant differences in baseline characteristics remained in the matched comparison group. Therefore, we believe that it is misleading and not appropriate to report the comparison results between the two unmatched groups.

Second, the matched vancomycin patients selected in the study were not representative of the general vancomycin population. This study started with the selected 3,708 vancomycin cases and 273 linezolid cases and further selected the qualified vancomycin $(n=621)$ and linezolid $(n=60)$ cases based on certain selection criteria. The final sample included the selected 60 vancomycin cases based on the propensity scores to match the 60 linezolid cases. Given this, the propensity score matching was not a randomized selection process, the selected vancomycin cases cannot represent the original 
vancomycin population $(n=621)$. There is a need to further examine the difference between the unselected/unmatched vancomycin cases $(n=561 ;[621-60=561])$ vs the 60 selected vancomycin cases. Any conclusions made about differences found between linezolid- and vancomycin-treated patients should be made and interpreted with caution.

Third, although clinical response rates were similar between the treatment groups, these response rates are considerably lower than those reported in previous retrospective or observational studies of patients with MRSA HAP or ventilator-associated pneumonia (VAP) $)^{2-4}$ and a prospective, randomized study conducted enrolling patients with grampositive infections in the People's Republic of China. ${ }^{5}$ In the study by Chan et al, clinical improvement defined by a clinical pulmonary infection score $\leq 6$ at day 3 of therapy was similar between linezolid- (63.0\%) and vancomycin (56.4\%)-treated patients with MRSA VAP. ${ }^{2}$ In the study by Watanabe et al, a response rate of $70.0 \%$ was reported for Japanese patients with MRSA HAP treated with linezolid. ${ }^{4}$ In the study by Peyrani et al, a response rate of $85 \%$ was reported for linezolid-treated patients compared to $69 \%$ for vancomycin-treated patients with MRSA VAP $(P=0.009)$. Response rates were also higher for linezolid than vancomycin in a randomized, double-blind, comparator-controlled, multicenter study enrolling 80 hospitalized patients with gram-positive pneumonia in the People's Republic of China. At end of treatment, the effective treatment rate was $78.6 \%$ for linezolid-treated patients and $52.9 \%$ for vancomycin-treated patients $(95 \%$ confidence interval, 2.99-48.3). ${ }^{5}$

The low response rate reported in this analysis potentially reflects the empirical administration of linezolid and vancomycin in this study. In general, HAP treatment was not guided by identification of a causative pathogen; of the 681 study patients, only five underwent bacterial culture testing. The authors noted that in Chinese tertiary care hospitals, routine assessment for the causative HAP pathogens is not commonly performed. The lower response rate reported by Song et al suggests that at least some vancomycin- and linezolid-treated patients had gram-negative HAP. Results from studies evaluating the microbiology of HAP in the People's Republic of China suggest, that gram-negative organisms are responsible for the majority of HAP and intensive care unit-acquired pneumonia cases. ${ }^{6,7}$

Although some patients in the study by Song et al received concomitant gram-negative therapy, it is not clear how many patients received active therapy against organisms such as Acinetobacter baumannii and Pseudomonas aeruginosa. In the matched patient cohort, significantly more patients receiving vancomycin than linezolid therapy received concurrent cephalosporin therapy (43.3\% vs $18.3 \%$; $P=0.007$ ). Depending on type of cephalosporin administered, potentially more patients treated with vancomycin than linezolid received therapy active against gram-negative organisms such as $P$. aeruginosa. Very little additional information on cephalosporin therapy is provided.

Fourth, we find it surprising that a similar response rate was noted in vancomycin-treated patients compared to linezolid-treated patients. Results from the most recently conducted Phase IV ZEPHyR study in patients with MRSA pneumonia showed a significantly higher response rate in patients treated with linezolid $(57.6 \%)$ than dose-adjusted vancomycin $(46.6 \% ; P=0.042) .{ }^{8}$ Of note, the clinical response rate with vancomycin therapy in the ZEPHyR trial was lower than that with linezolid therapy even though the vancomycin dose was higher than that utilized in the study by Song et al. In the ZEPHyR trial, vancomycin therapy was initiated at a dose of $15 \mathrm{mg} / \mathrm{kg}$ as recommended by current guidelines ${ }^{9}$ and adjusted based on trough levels. In contrast, the mean vancomycin dose in the study by Song et al was only approximately $1,500 \mathrm{mg}$ per day. Potential explanations for this result include a lack of MRSA pneumonia in study participants and the significant differences in baseline characteristics that remained between the treatment groups following propensity score matching.

Fifth, Song et al reported a significantly lower in-hospital mortality rate for vancomycin-treated patients compared to linezolid-treated patients $(3.3 \%$ vs $18.3 \% ; P=0.013)$. This difference in mortality rate was lost after adjustment for an imbalance in variables between the matched treatment groups (OR: 0.186; 95\% confidence interval: 0.033-1.39; $P=0.055$ ). No mortality differences were found between linezolid- and vancomycin-treated patients in the ZEPHyR trial and several meta-analyses published since the beginning of the year 2010. ${ }^{10-14}$ Song et al however concludes that "vancomycin likely had a lower in-hospital mortality rate when compared with linezolid." Caution must be exercised when making comparisons between the linezolid and vancomycin treatment groups as statistically significant imbalances in baseline characteristics could account for the increased mortality reported in patients treated with linezolid compared to vancomycin. No differences in mortality were found after accounting for differences in baseline patient characteristics that remained following propensity-score matching.

Lastly, Song et al reported significantly lower acquisition costs for vancomycin (RMB 2,880) than linezolid therapy (RMB 8,194; $P<0.001$ ). Lower vancomycin acquisition costs were also reported in a cost-effectiveness analysis of 
linezolid vs vancomycin therapy for patients with MRSAconfirmed nosocomial pneumonia using cost data from four cities in the People's Republic of China (ie, Beijing, Guangzhou, Nanjing, and Xi'an). ${ }^{15}$ Although acquisition costs were significantly higher for linezolid than vancomycin in the study by Song et al, no difference in median total hospital costs was found $(P=0.076)$. Similarly, results from the cost-effectiveness analysis in all four Chinese cities showed that linezolid was a cost-effective alternative to vancomycin in patients with MRSA-confirmed nosocomial pneumonia having similar or lower total treatment costs.

In conclusion, we challenge the statement made by Song et al that "future clinical practice guidelines could use this evidence to further support the established first-line treatment with vancomycin for difficult HAP.” Additional studies are needed in the People's Republic of China to determine the causative pathogens in patients with HAP. Vancomycin and linezolid should only be administered to patients with a high suspicion of or documented MRSA pneumonia. In patients with MRSA HAP, results from randomized clinical trials suggest similar or increased response rates for linezolid compared to vancomycin therapy ${ }^{5,8}$ with no difference in mortality. ${ }^{8,10-14}$ Furthermore, results from a cost-effectiveness analysis conducted using data from Beijing, Guangzhou, Nanjing, and Xi' an show that linezolid compared to vancomycin therapy in patients with MRSA nosocomial pneumonia is a cost-effective therapy.

\section{Disclosure}

BAL and XG are employees of Pharmerit International which received funding from Pfizer Investment Co, Ltd. ZL has served as a speaker for Pfizer Investment Co, Ltd. YC is an employee of Pfizer Investment Co, Ltd. The authors report no other conflicts of interest in this communication.

\section{References}

1. Song Y, Yang Y, Chen W, et al. Clinical response and hospital costs associated with the empirical use of vancomycin and linezolid for hospitalacquired pneumonia in a Chinese tertiary care hospital: a retrospective cohort study. Clinicoecon Outcomes Res. 2014;6:451-461.
2. Chan JD, Pham TN, Wong J, et al. Clinical outcomes of linezolid vs vancomycin in methicillin-resistant Staphylococcus aureus ventilatorassociated pneumonia: retrospective analysis. J Intensive Care Med. 2011;26(6):385-391.

3. Peyrani P, Wiemken TL, Kelley R, et al. Higher clinical success in patients with ventilator-associated pneumonia due to methicillinresistant Staphylococcus aureus treated with linezolid compared with vancomycin: results from the IMPACT-HAP study. Crit Care. 2014;18(3):R118.

4. Watanabe A, Goto H, Soma K, et al. Usefulness of linezolid in the treatment of hospital-acquired pneumonia caused by MRSA: a prospective observational study. J Infect Chemother. 2012;18(2):160-168.

5. Lin DF, Zhang YY, Wu JF, et al. Linezolid for the treatment of infections caused by Gram-positive pathogens in China. Int $J$ Antimicrob Agents. 2008;32(3):241-249.

6. Liu YN, Cao B, Wang H, et al. [Adult hospital acquired pneumonia: a multicenter study on microbiology and clinical characteristics of patients from 9 Chinese cities]. Zhonghua Jie He He Hu Xi Za Zhi. 2012;35(10):739-746. Chinese.

7. Zhang Y, Yao Z, Zhan S, et al. Disease burden of intensive care unit-acquired pneumonia in China: a systematic review and meta-analysis. Int J Infect Diss. 2014;29:84-90.

8. Wunderink RG, Niederman MS, Kollef MH, et al. Linezolid in methicillin-resistant Staphylococcus aureus nosocomial pneumonia: a randomized, controlled study. Clin Infect Dis. 2012;54(5):621-629.

9. Liu C, Bayer A, Cosgrove SE, et al. Clinical practice guidelines by the infectious diseases society of america for the treatment of methicillinresistant Staphylococcus aureus infections in adults and children: executive summary. Clin Infect Dis. 2011;52(3):285-292.

10. Jiang $H$, Tang RN, Wang J. Linezolid versus vancomycin or teicoplanin for nosocomial pneumonia: meta-analysis of randomised controlled trials. Eur J Clin Microbiol Infect Dis. 2013;32(9):1121-1128.

11. Kalil AC, Klompas M, Haynatzki G, Rupp ME. Treatment of hospitalacquired pneumonia with linezolid or vancomycin: a systematic review and meta-analysis. BMJ Open. 2013;3(10):e003912.

12. Kalil AC, Murthy MH, Hermsen ED, Neto FK, Sun J, Rupp ME. Linezolid versus vancomycin or teicoplanin for nosocomial pneumonia: a systematic review and meta-analysis. Crit Care Med. 2010;38(9): 1802-1808.

13. Walkey AJ, O'Donnell MR, Wiener RS. Linezolid vs glycopeptide antibiotics for the treatment of suspected methicillin-resistant Staphylococcus aureus nosocomial pneumonia: a meta-analysis of randomized controlled trials. Chest. 2011;139(5):1148-1155.

14. Wang Y, Zou Y, Xie J, et al. Linezolid versus vancomycin for the treatment of suspected methicillin-resistant Staphylococcus aureus nosocomial pneumonia: a systematic review employing meta-analysis. Eur J Clin Pharmacol. 2015;71(1):107-115.

15. Tan SC, Wang X, Wu B, et al. Cost-effectiveness of linezolid versus vancomycin among patients with methicillin-resistant Staphylococcus aureus confirmed nosocomial pneumonia in China. Value Health Regional Issue. 2014:94-100. 


\section{Authors' reply}

\author{
Yuanlin Song' \\ Yicheng Yang ${ }^{2}$ \\ Wendong Chen 3,4 \\ Wei Liu \\ Kai Wang ${ }^{2}$ \\ Xuehai $\mathrm{Li}^{5}$ \\ Ke Wang ${ }^{2}$ \\ Manny Papadimitropoulos ${ }^{3,6}$ \\ William Montgomery ${ }^{7}$
}

'Department of Pulmonary Medicine, Zhongshan Hospital, Fudan University, Shanghai, People's Republic of China; ${ }^{2}$ Lilly Suzhou Pharmaceutical Co, Ltd, Shanghai Branch, Shanghai, People's Republic of China; ${ }^{3}$ Division of Social and Administrative Pharmacy, Leslie Dan Faculty of Pharmacy, University of Toronto, Toronto, ON, Canada; ${ }^{4}$ Normin Health, Toronto, ON, Canada; ${ }^{5}$ VitalStrategic Research Institute, Shanghai, People's Republic of China; ${ }^{6} \mathrm{Global}$ Health Outcomes Research, Eli Lilly, Indianapolis, IN, USA; ${ }^{7}$ Eli Lilly Australia Pty Ltd, West Ryde, NSW, Australia

Correspondence: William Montgomery

Eli Lilly Australia Pty Ltd, I 12 Wharf Rd,

West Ryde, NSW 2064, Australia

Tel +6I 293254335

Fax +6I 293254334

Email montgomery_bill@lilly.com

\section{Dear editor}

We would like to thank Lesher et al for the comments on our recent publication that examined the clinical response and hospital costs associated with the empirical use of vancomycin and linezolid for hospital-acquired pneumonia (HAP) in a Chinese tertiary care hospital. ${ }^{1}$ Whilst we concur with a number of the points made by the authors, there are a number that deserve to be challenged. Our study analyzed realworld outcomes of patients treated with one of two different antibiotics, vancomycin or linezolid, who were diagnosed with HAP in a tertiary care hospital in Shanghai, People's Republic of China. A number of the limitations discussed by Lesher et al highlight the differences between research conducted using real-world data and randomized studies.

Firstly, the criticism that the two treatment groups compared differed significantly in terms of baseline characteristics even after we had taken steps to create matched pairs of patients using one-to-one propensity score matching is not accurate. In our study, several variables were not well balanced between the matched treatment groups. However, we conducted multiple regression analyses to adjust the unbalanced variables in the matched patients to overcome this limitation. The results, after the regression adjustment, were highly consistent with the results of the direct comparisons between the matched treatment groups. Thus, we believe that the confounding effects associated with these unbalanced variables on the measured outcomes in this study were modest at best, and that appropriate steps were taken to account for most differences.

Secondly the criticism that the vancomycin patients included in the matched comparison were not representative of the overall sample of vancomycin patients is unfounded. In our study, the number of patients receiving linezolid was much smaller than the number of patients receiving vancomycin (273 vs 3,708). The main purpose of employing a matching algorithm was to compare the two antibiotic treatments across comparable groups of patients. To do this we used one-to-one propensity score matching to identify patients who had similar baseline characteristics across both treatment arms. Using this approach we believe provided findings that are more applicable to the patients who are likely to receive linezolid for the treatment of HAP under normal clinical conditions.

We note that the response rates seen in our study are considerably lower than those reported in some previous studies. Differences in findings between studies in the same therapeutic area are a typical phenomenon associated with clinical research due to many reasons, including differences in study design, treatment patterns, clinical settings, patient characteristics, and inclusion/exclusion criteria. The treatment response rates cited by Lesher et al are mainly from randomized trials and studies that included patients with different characteristics to those in our study who were from a real-world clinical setting in the People's Republic of China. Thus, the variances in treatment response rates between our study and the other studies are not unexpected. Our study findings are well interpreted in our paper by emphasizing that the treatment used was empirical, and most of our patients were not tested for causative pathogens. It is inappropriate to compare the results from our study to those from a prospective randomized study designed to test efficacy. We agree that the lower treatment response rates in our study cohort could be the result of a higher proportion of HAP caused by gram-negative bacteria, which may explain the higher mortality observed in patients receiving linezolid in our study. As pointed out by Lesher et al, previous research from the People's Republic of China has identified high rates of gram-negative bacteria in patients with HAP. ${ }^{2}$ Significantly more patients receiving vancomycin than linezolid therapy received concurrent cephalosporin therapy; however, the unbalanced concurrent cephalosporin use was further adjusted in the regression analysis in the matched patients. 
Thus, the confounding effects associated with the concurrent cephalosporin use were mainly related to the differences in the distribution of cephalosporin drugs associated with the two treatments. The results of our study findings support the high risk of gram-negative bacterial infection associated with HAP, in this clinical setting.

The comparison of the results from our study to those from studies conducted in different countries and using different study designs highlights one of the pitfalls when attempting to make cross-study comparisons. The main purpose of our study was to observe naturalistic treatment patterns and outcomes in a real-world setting in a tertiary care hospital in the People's Republic of China. In the ZEPHyR study, ${ }^{3}$ the superiority findings associated with linezolid were based on the analysis of the per-protocol group, whereas the analysis based on intention-to-treatment basis found highly comparable treatment response for the two antibiotic treatments. Additionally, the rate of vancomycin treatment in the ZEPHyR study was lower because serum vancomycin levels did not reach the optimal levels needed for adequate penetration into the lung and therefore the efficacy of vancomycin was likely to have been underestimated in this study. ${ }^{3}$ Subsequently, two published meta-analyses did not identify any superiority for linezolid over vancomycin for the treatment of pneumonia. ${ }^{4,5}$

Our study's findings can be used to support treatment decision making in real-world hospital settings in the People's Republic of China, where most patients with HAP have no causative pathogen identified, and where empirical antibiotic treatment is routine practice.

Our study reported a significantly lower in-hospital mortality rate associated with vancomycin treatment compared to linezolid treatment. The observed higher mortality associated with linezolid treatment in our study could reflect the high risk of gram-negative infection in Chinese patients with HAP, previously discussed.

Because the patient cohorts, clinical settings, treatment patterns, and health care resource use patterns observed in our study were likely different from those in the costeffectiveness analyses cited by Lesher et al, it is inappropriate to apply the conclusions of these studies to our findings. Even if we assume that the two treatments were associated with similar costs in our study, the increased in-hospital mortality associated with linezolid treatment does not support a cost-effectiveness argument for linezolid when compared with vancomycin. On the other hand, the cost-effectiveness analyses referred to in this letter used information on health care resource and clinical outcomes directly obtained from a study conducted in the USA and European centers where it is expected that clinical practices and treatment patterns would likely be very different to those in the People's Republic of China. We believe the results and conclusion from this comparison may include some biases, and it is therefore inappropriate to use it to critique our study.

In summary, we thank the authors of this letter by providing us with an opportunity to further clarify the interpretation of our study's findings and guide the use of our evidence when empirically treating patients with HAP. However, the evidence cited by Lesher et al are mainly based on studies including patients with confirmed MRSA HAP. As such their opinions may not be relevant to real-world clinical settings, where HAP is frequently treated empirically without the benefit of identification of the causative pathogen.

\section{Disclosure}

Yuanlin Song received honorariums for travel and expenses of the study. Wei Liu, Ke Wang, Manny Papadimitropoulos, and William Montgomery are employees of Eli Lilly. Contributions from Yicheng Yang and Kai Wang were made whilst they were employees of Eli Lilly. Wendong Chen and Xuehai Li received a project consulting fee from Eli Lilly to compensate for their time on the project. The authors report no other conflicts of interest in this communication.

\section{References}

1. Song Y, Yang Y, Chen W, et al. Clinical response and hospital costs associated with the empirical use of vancomycin and linezolid for hospitalacquired pneumonia in a Chinese tertiary care hospital: a retrospective cohort study. Clinicoecon Outcomes Res. 2014;6:451-461.

2. Liu YN, Cao B, Wang H, et al. [Adult hospital acquired pneumonia: a multicenter study on microbiology and clinical characteristics of patients from 9 Chinese cities]. Zhonghua Jie He He Hu Xi Za Zhi. 2012;35(10):739-746. Chinese.

3. Kalil AC. Linezolid does not show advantages over vancomycin in modulating the pulmonary immune response: how should we conciliate these new findings with the Zephyr trial results? Crit Care Med. 2011;39(8):2009-2010.

4. Wang Y, Zou YM, Xie J, et al. Linezolid versus vancomycin for the treatment of suspected methicillin-resistant Staphylococcus aureus nosocomial pneumonia: a systematic review employing meta-analysis. Eur J Clin Pharmacol. 2015;71(1):107-115.

5. Kalil AC, Klompas M, Haynatzki G, et al. Treatment of hospital-acquired pneumonia with linezolid or vancomycin: a systematic review and metaanalysis. BMJ Open. 2013;3(10):e003912. 
Dove Medical Press encourages responsible, free and frank academic debate. The content of the ClinicoEconomics and Outcomes Research 'letters to the editor' section does not necessarily represent the views of Dove Medical Press, its officers, agents, employees, related entities or the ClinicoEconomics and Outcomes Research editors. While all reasonable steps have been taken to confirm the content of each letter, Dove Medical Press accepts no liability in respect of the content of any letter, nor is it responsible for the content and accuracy of any letter to the editor.

ClinicoEconomics and Outcomes Research

Dovepress

\section{Publish your work in this journal}

ClinicoEconomics \& Outcomes Research is an international, peerreviewed open-access journal focusing on Health Technology Assessment, Pharmacoeconomics and Outcomes Research in the areas of diagnosis, medical devices, and clinical, surgical and pharmacological intervention. The economic impact of health policy and health systems script management system is completely online and includes a very quick and fair peer-review system, which is all easy to use. Visit http://www.dovepress.com/testimonials.php to read real quotes from published authors.

Submit your manuscript here: http://www.dovepress.com/clinicoeconomics-and-outcomes-research-journal 